\title{
Expression and clinical implications of basic leucine zipper ATF-like transcription factor 2 in breast cancer
}

Yingying Lin ${ }^{1}$, Xusheng Zhou ${ }^{1}$, Wei Peng ${ }^{1}$, Jing Wu ${ }^{1}$, Xiufeng Wu ${ }^{2^{*}}$, Yan Chen ${ }^{1 *}$ and Zhaolei Cui ${ }^{1 *}$

\begin{abstract}
Background: Basic leucine zipper ATF-like transcription factor 2 (BATF2) has been reported to participate in the occurrence and development of some malignancies. Herein, we aimed to explore the expression pattern and clinical implications of BATF2 in breast cancer (BC).
\end{abstract}

Methods: We assessed the differences in BATF2 mRNA expression between cancerous and noncancerous tissues in BC using GEPIA and UALCAN data and in BATF2 protein expression pattern using Human Protein Atlas (HPA) data. BATF2 co-expression networks were analyzed in Coexpedia. The association between the differentially expressed BATF2 mRNA and BC prognosis was assessed using UALCAN, OSbrca, and GEPIA databases. In external validations, BATF2 protein expression in BC tissues was quantitated using a tissue microarray and immunohistochemistry $(\mathrm{IHC})$ analysis, and BATF2 mRNA expression in serum and serum-derived exosomes of BC patients using real-time quantitative reverse transcription polymerase chain reaction (qRT-PCR).

Results: No difference in the BATF2 mRNA expression level was found between cancerous and noncancerous tissues in BC based on databases. There were low-to-moderate levels of increases in BATF2 protein expressions in $B C$ cases from the HPA cohort. BATF2 mRNA expression was negatively correlated with androgen receptor (AR) and positively correlated with BRCA2 DNA repair associated (BRCA2), marker of proliferation Ki-67 (Mki67), and tumor protein p53 (TP53) expressions. Generally, BATF2 mRNA exhibited a non-significant association with BC prognosis; yet the subgroup analyses showed that triple-negative breast cancer (TNBC) patients with high BATF2 mRNA expressions had a longer overall survival (OS). Our IHC analysis revealed a positive rate of BATF2 protein expression of $46.90 \%$, mainly located in the nucleus of cancer cells in BC, and the OS of BC patients with high BATF2 protein expressions was prolonged. The positive rates of BATF2 mRNA expressions in the serum and exosomes were 45.00 and $41.67 \%$, respectively. Besides, the AUCs of serum and exosomal BATF2 mRNA for BC diagnosis were 0.8929 and 0.8869 , respectively.

\footnotetext{
*Correspondence: wxf200104@hotmail.com; chenyan220422@fjmu.edu.cn; cuileidizi@fjmu.edu.cn

${ }^{2}$ Department of Breast Surgical Oncology, Fujian Medical University Cancer Hospital, Fuzhou, Fujian, China

'Laboratory of Biochemistry and Molecular Biology Research, Department of Clinical Laboratory, Fujian Medical University Cancer Hospital, No. 420 Fuma Road, Jin'an District, Fuzhou 350014, Fujian Province, China
} 
Conclusions: BC patients exhibit low-to-moderate expressions in BATF2 mRNA expression levels in cancerous tissues. The high BATF2 protein expression can be a potential indicator of a better BC prognosis. Serum and exosomal BATF2 mRNA levels also serve as promising noninvasive biomarkers for BC diagnosis.

Keywords: BATF2, Breast cancer, Bioinformatics, Serum, Exosomes, Biomarker

\section{Background}

$\mathrm{BC}$ is one of the most prevalent malignancies in women [1]. The latest cancer statistics showed that BC topped the list of cancer morbidity in Chinese women, with a mortality rate ranking fourth [2]. Most studies agree that intricate multi-gene networks are involved in the occurrence, development, and metastasis of $\mathrm{BC}$ in synergy, accompanied by mutations and/or abnormal genetic activities, such as proto-oncogene activation, apoptosis inhibition of tumor suppressor genes, abnormalities in gene expressions [3-5]. For personalized diagnosis, treatment, and prognosis evaluation of $\mathrm{BC}$, arduous tasks are ahead of us to unveil the underlying genetic mechanisms behind the onset and evolution of $\mathrm{BC}$.

$B A T F-2$, also known as suppressor of activator protein-1 regulated by interferon (SARI), is a recently discovered tumor suppressor gene using subtractive hybridization in 2008 [6]. It is a type I interferon (IFN) inducible protein with a leucine zipper and an activator protein (AP)-1 transcription factor family member and has specific structural characteristics to activate transcription factors [6]. Pieces of evidence show that the BATF-2 gene, though expressed in a variety of normal tissue cells (e.g., melanocytes, astrocytes, pancreatic mesothelial cells, and prostate epithelial cells), can selectively inhibit the growth of tumor cells $[6,7]$. Strikingly, recent studies found its roles in the occurrence and development of various malignancies. For example, BATF-2 mRNA expression is down-regulated in chronic myeloid leukemia (CML) patients compared to healthy individuals, and BCR-ABL chimeric protein participates in the inhibition of BATF-2 gene expression [8]. Abnormalities in BATF-2 and cellular communication network factor 1 (CCN1) expressions and their correlation are closely associated with malignant behaviors of colorectal cancer cells, affecting the prognosis of patients $[9,10]$. Moreover, in other tumors such as hepatocellular carcinoma (HCC) [11], non-small-cell lung cancer (NSCLC) [12], esophageal squamous cell carcinoma (ESCC) [13] and gastric cancer (GC) [14], the down-regulation of BATF-2 expression is associated with a poor prognosis. Wang et al. found that BATF-2 regulated the epithelialmesenchymal transition (EMT) and lung adenocarcinoma (LUAD) metastasis [15]. However, little is known about the roles of BATF-2 in BC. Studies on the expression patterns and clinical implications of BATF-2 in BC are needed.
In contrast to preclinical and clinical studies that are time-consuming, bioinformatics has provided convenience or high efficiency for studies of genetic activity in cancers since the Human Genome Project paved the way [16]. As gene and protein reaction networks consist of voluminous interactions, bioinformatics is a useful tool for the studies of genomics, proteomics, and other fields [17]. In this work, we sought to explore the expression patterns and diagnostic and prognostic implications of BATF2 mRNA and protein expressions in $\mathrm{BC}$ using bioinformatics, which were subsequently verified in the serum, serum-derived exosomes, and cancer tissues of BC patients using qRT-PCR and IHC.

\section{Methods}

\section{Study design}

We first assessed the difference in BATF2 mRNA expression between cancerous and noncancerous tissue samples from GEPIA [18] and UALCAN [19] databases, and that in BATF2 protein expression between the two cohorts of samples in HPA. The gene co-expression network of BATF2 was plotted using Coexpedia [20], and the correlations between BATF2 mRNA and other coexpressed markers (e.g., HER2 and BRCA2) in BC were evaluated using GEPIA. The prognostic value of BATF2 mRNA expression in $\mathrm{BC}$ prognosis was assessed using OSbrca [21], UALCAN, and Kaplan-Meier Plotter [22]. Based on the bioassay data, we further set up tissue microarray and IHC analysis to verify the BATF2 protein expression pattern in the serum and serum-derived exosomes of BC patients and its correlation with clinicopathological features and prognosis. Serum and exosomal BATF2 mRNA expressions were determined using qRT-PCR analysis.

\section{Clinical data}

We performed protein expression analysis of BATF2 using clinical data of a high-throughput tissue microarray (HBreD145Su02, SHANGHAI OUTDO BIO$\mathrm{TECH}$ ) consisting of 145 cancer tissues and 90 adjacent noncancerous tissues (SHANGHAI OUTDO BIOTECH) from 145 female patients who were pathologically diagnosed and underwent radical mastectomy from August 2004 to December 2008. Their medical records were complete and available. These patients were aged from 33 to 88 years, with an average age of 58 years. As for $\mathrm{BC}$ subtypes, one case was diagnosed with intraductal 
carcinoma, 2 with mucinous carcinoma, 4 with invasive lobular carcinoma, 119 with invasive ductal carcinoma, 9 with invasive micropapillary carcinoma, 3 with invasive lobular carcinoma, 2 with medullary carcinoma, and 5 with mucinous carcinoma. These patients did not receive radiotherapy, chemotherapy, or other tumorrelated treatments before the operation. Also, 60 serum samples of BC patients hospitalized in Fujian Cancer Hospital from December 2018 to May 2019 were collected, and 56 serum samples of normal healthy people were collected as controls. All included BC individuals were invasive ductal carcinoma and non-TNBC (triplenegative breast cancer) cases, with an average age of 56 years. All serum specimens were collected with the approval of the Ethics Committee of Fujian Medical University Cancer Hospital (ethical approval certificate: No. SQ2018-015-01).

\section{Public data and bioinformatics tools}

GEPIA is a tumor analysis webserver for assessing mRNA expression data, other gene expression profiles, differentially expressed genes and survival of patients with various tumors and their subtypes using TCGA and GTEx data [18]. UALCAN database offers gene expression analysis and survival analysis based on clinical data from TCGA [19]. In this study, both GEPIA and UALCAN databases were utilized to appraise the difference in BATF2 mRNA expression between cancerous and noncancerous tissues in BC. HPA database provides information on the tissue and cell distribution of 26,000 human proteins and the expression of each protein in 64 cell lines across 48 human normal tissues and 20 tumor tissues. We extracted data about the expression of BATF2 protein and clinicopathological features of $\mathrm{BC}$ tissues from HPA. The gene co-expression network of $B A T F 2$ together with other target genes was depicted using Coexpedia [20] to predict relevant diseases or pathways enriched in target genes. OSbrca a professional prognostic tool with comprehensive data sources and large sample size (2277 malignancies and 31,310 patients from 209 data sets derived from TCGA, GEO, CGGA, and other databases). Kaplan-Meier Plotter to evaluate mRNA, miRNA, and protein expressions on survival supports the multi-gene query and pan-cancer analysis of 21 tumors. We analyzed the correlations between BATF2 mRNA expression and BC prognosis using OSbrca [21], Kaplan-Meier Plotter [22], GEPIA, and UALCAN databases and appraised its prognostic effect based on the prognosis-survival curve.

\section{IHC analysis}

The BATF2 protein in paraffin-embedded BC tissues was identified by the rabbit anti-human BATF2 polyclonal antibody (Abcam, Catalog No.ab204510; 1:50) using the EliVisionTM Plus two-step detection system according to its protocol. PBS was used instead of the primary antibody as a negative control. The experimental results were judged to be completed under the guidance of clinically experienced pathologists. The scoring criteria were based on our previously published articles $[23,24]$.

\section{Isolation and identification of exosomes}

Exosomes were extracted from serum samples using an exoRNeasy Serum/Plasma Midi Kit (QIAGEN, Catalog No.77044), as described in the manufacturer's protocol (www.qiagen.com/hb-1179). The extracted exosome vesicles were determined by transmission electron microscopy (TEM). As for the quantification of BATF2 protein expression, samples were lysed by RIPA lysis to extract the total protein. The equivalent amount of sample proteins were loaded onto each well for western blot analysis, and samples were incubated with mouse antihuman GAPDH monoclonal antibody (1:1000) and rabbit anti-human monoclonal CD63 (Abcam, Catalog No.ab217345) and CD9 (Abcam, Catalog No.ab92726) primary antibodies (1:100), visualized and photographed. According to The International Society for the extraction of extracellular vesicles [25], the rabbit anti-human Cytochrome C monoclonal antibody (Beyotime, Catalog No.AF2047; 1:100) was utilized as a negative control. The specific procedures had been described in our previous study series $[23,24]$.

\section{Real-time quantitative reverse transcription polymerase chain reaction (qRT-PCR)}

The total RNA from the serum and serum-derived exosomes was extracted using a miRNeasy Kit and exoRNeasy Serum/Plasma Midi Kit according to the manufacturer's protocol. The extracted total RNA was reverse transcribed into cDNA using the Transcriptor First-strand cDNA synthesis kit (Roche). The quantitative real-time PCR analysis was performed in the ABI7500 fluorescence quantitative PCR detector using SYBR Green Master (ROX) Mix. The primer sequences of the target products incorporated $B A T F 2-\mathrm{F}$ : 5'-GCCTAAGCCATGCACCTCTGT-3', BATF2-R: '-TCTTCAGCTGCCTTTGTTGCTC -3', GAPDH-F: 5'-GGAGCGAGATCCCTCCAAAAT-3', and GAPD H-R: 5' -GGCTGTTGTCATACTTCTCATGG-3' [23, 24]. The amplification was carried out as follows: initial denaturation at $95^{\circ} \mathrm{C}$ for $10 \mathrm{~min}, 40$ cycles of amplification at $95^{\circ} \mathrm{C}$ for $15 \mathrm{~s}$, and extension at $60^{\circ} \mathrm{C}$ for $1 \mathrm{~min}$. The expression level of BATF2 mRNA relative to the internal reference gene was evaluated using the $2^{-\Delta \Delta C T}$ method. 


\section{Statistical analysis}

SPSS 16.0 software was employed for all statistical analyses. The relative experimental values were expressed as mean \pm standard deviation (SD). After the homogeneity test for variance, the comparisons of differences between groups were accomplished using the Student's t-test, and two-side $P<0.05$ was assumed as statistical significance.

\section{Results}

Patterns of BATF2 mRNA and protein expressions in BC All procedures of this study design were depicted in Fig. 1. The pan-cancer analysis showed that the expression level of BATF2 mRNA in breast invasive carcinoma (BRCA) was slightly lower than that in normal controls, but without a significant difference (Fig. 2A and B). Comparisons of BATF2 mRNA expression between distinct cancer stages, histologic subtypes, major subclasses, and menopause versus non-menopause status in BRCA were respectively assessed and summarized in Fig. 1C-G. Specifically, BATF2 mRNA expression pronouncedly increased in patients with TNBC-IM $(n=20)$ (Fig. 2F), or medullary BC (Fig. 2G). Besides, BATF2 protein expression (using HPA data) also detectable in cancer cell lines and cancer tissues (Fig. 3A and B), which was tested positive in more than $58.33 \%(7 / 12)$ of the BRCA patients in the HPA data. Among the positive samples of the HPA data, a moderate level (Staining: medium; Intensity: moderate; Quantity: > 75\%) of BATF2 protein expression appeared in 5 cases, and a low level (Staining: low; Intensity: weak; Quantity: 75-25\%) of BATF2 expression was shown in 2 cases (Fig. 3C-F shows the expression status of BATF2 in 4 BRCA cases).

\section{BATF2 interaction network and correlation analysis}

The gene co-expression network of BATF2 mRNA in Pan-cancer was plotted using Coexpedia, as shown in Fig. 4A. Finally, 98 genes were predicted to interact with $B A T F 2 \mathrm{mRNA}$, and the co-expression of BATF2 with TAP1, STAT1, and PSMB9 was the most critical in the occurrence and development of BC (Fig. 4B). Based on GEPIA database, BATF2 mRNA expression was negatively correlated with $A R$ expression (Fig. 4C) and positively associated with BRCA2 (Fig. 4D), Mki67 (Fig. 4E), and TP53 (Fig. 4F) expressions (all with $P<0.01$ ). There were nonsignificant associations between $B A T F 2$ expression and HER2, EGFR, TP73, or AFP (Fig. 4G-J) (all with $P>0.05)$.

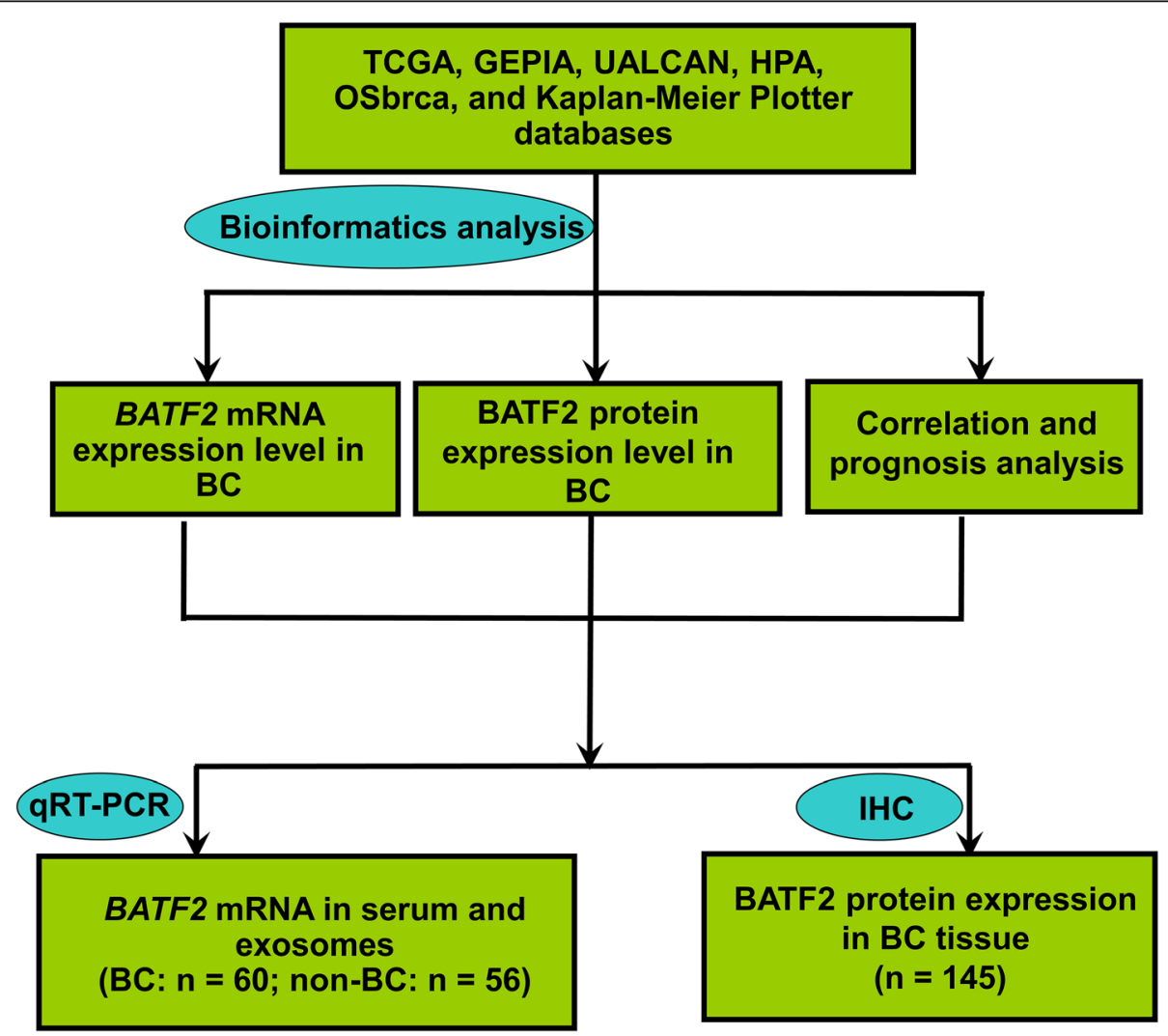

Fig. 1 Flow chart of the study design 
Lin et al. BMC Cancer

(2021) 21:1062

Page 5 of 14

A

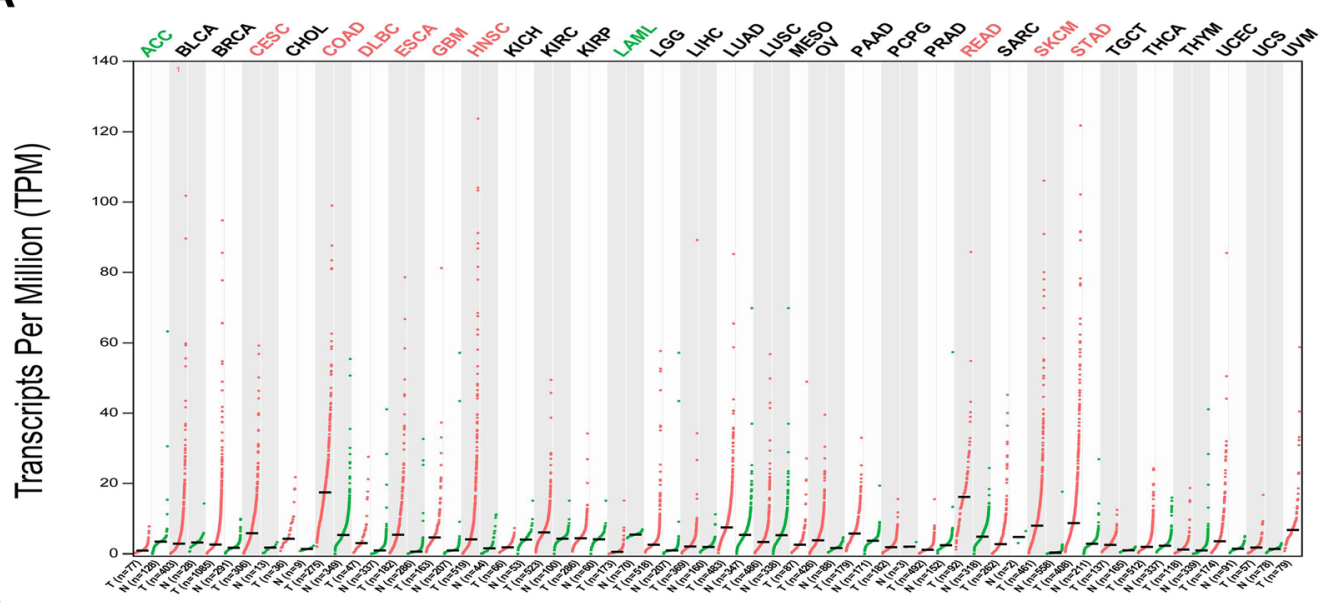

B Expression of BATF2 across TCGA cancers (with tumor and normal samples)

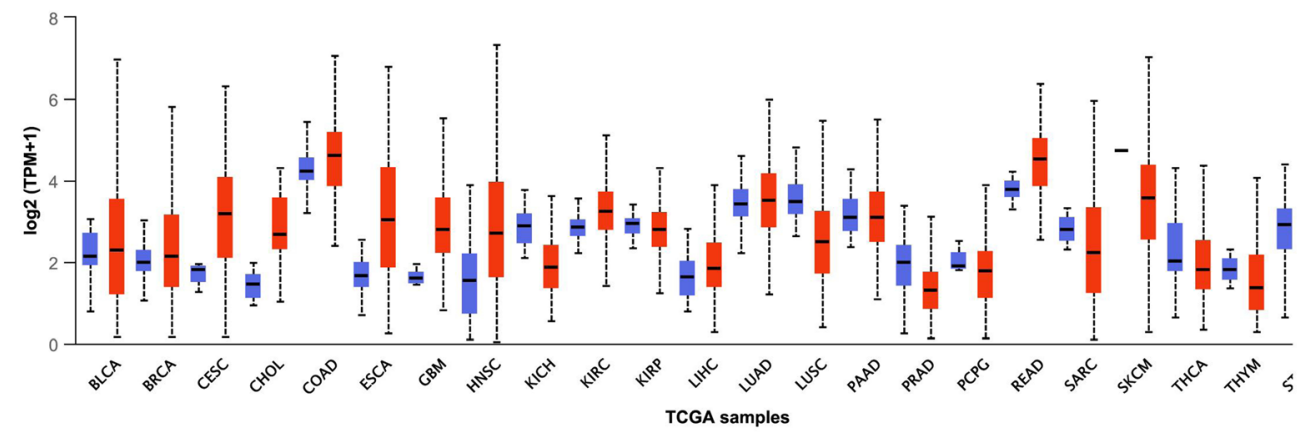

C

Session of BATF2 in BRCA based on Sample ones
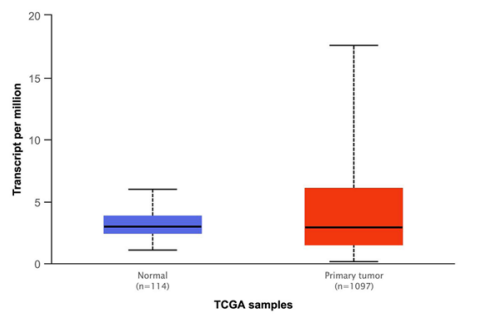

D
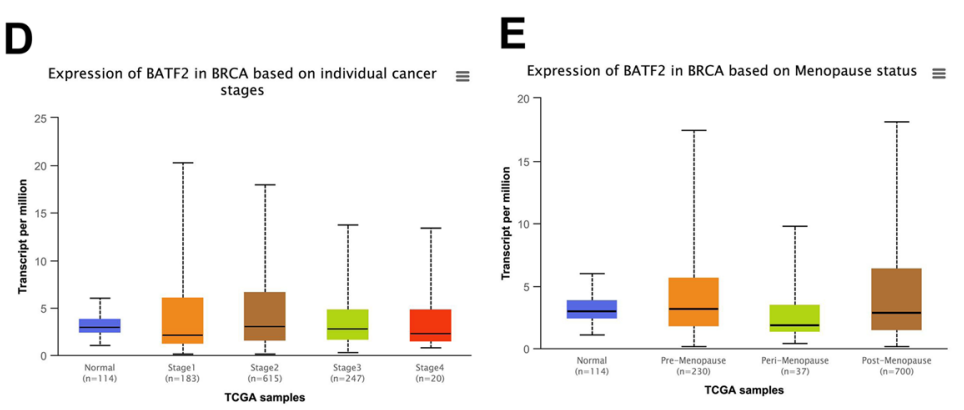

$\mathbf{F}$

Expression of BATF2 in BRCA based on Major subclasses (with TNBC $\equiv$

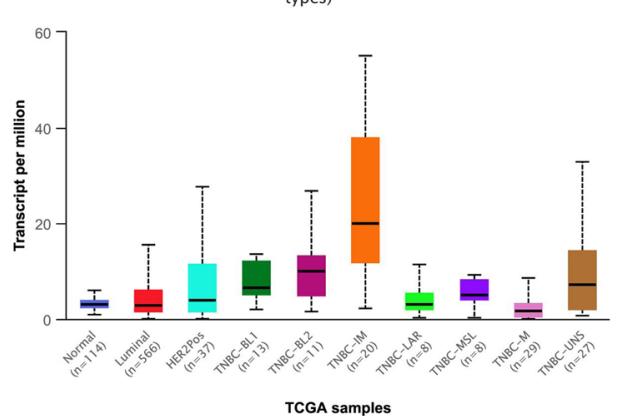

G

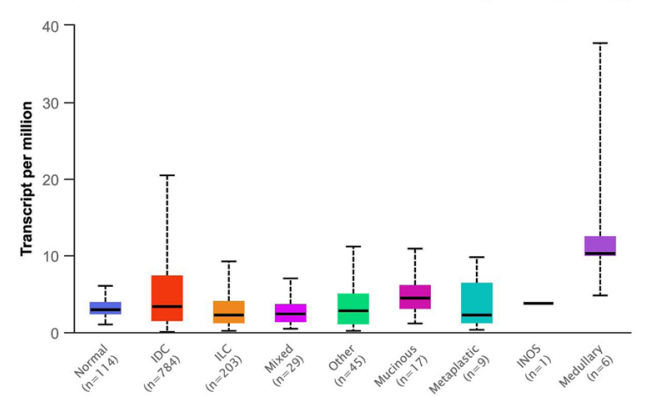

Fig. 2 Expressions of BATF2 RNA in cancers analyzed based on GEPIA and UALCAN databases. Pan-cancer view of BATF2 mRA in (A) GEPIA and (B) UALCAN databases. Expressions of BATF2 RNA in BRCA cases based on (C) sample types, (D) cancer stages, (E) menopause status, $(\mathbf{F}$ subclasses, and (G) histologic subtypes 


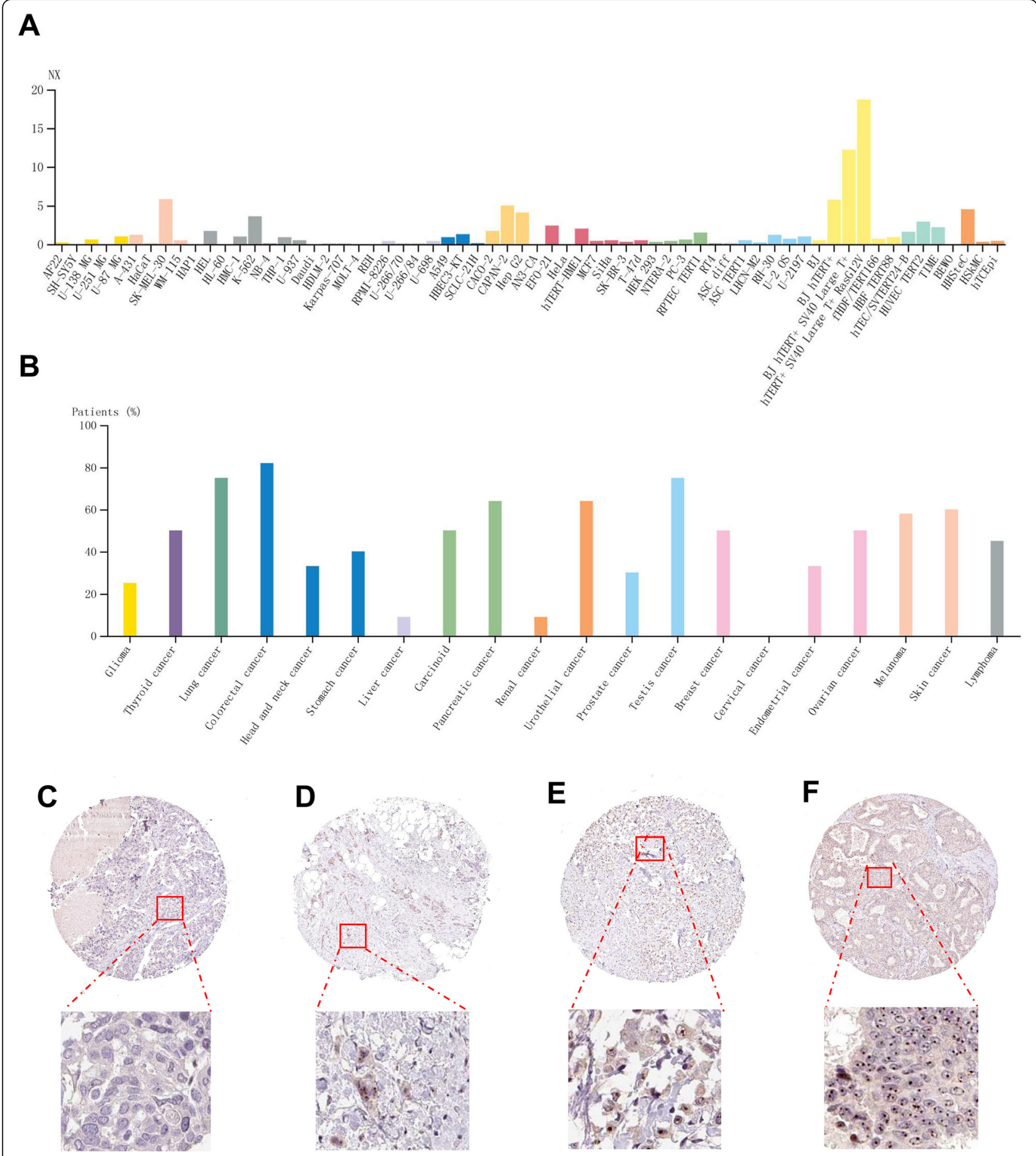

Fig. 3 Expressions of BATF2 mRNA and protein in cancers analyzed based on HPA database. (A) Pan-cancer view of BATF2 mRNA in cell lines. (B) Pan-cancer view of BATF2 protein expression in cancer patients. (C-F) Expression of BATF2 in BC tissues by IHC $(\times 400)$

BATF2 mRNA expression has little influence on BC prognosis

Based on different datasets in OSbrca, BATF2 mRNA expression displayed no significant influence on $\mathrm{BC}$ prognosis (Fig. 5A-C). However, the subgroup analysis showed that the overall survival (OS) of TNBC patients with a high $B A T F 2$ mRNA level was significantly longer than that of patients with a low BATF2 mRNA level $(P=0.0485$; Fig. 5D). Based on UALCAN datasets, high $B A T F 2$ expression level and menopause status were 


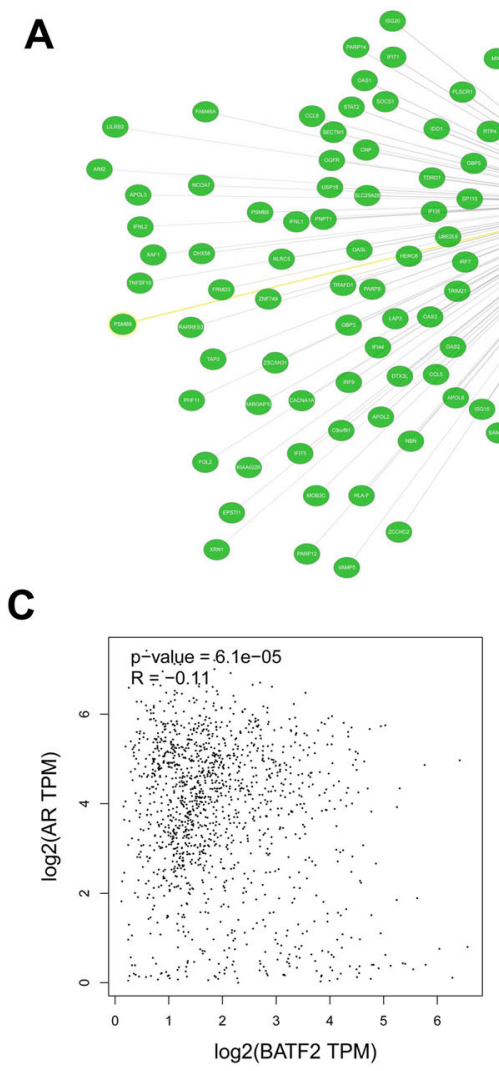

D

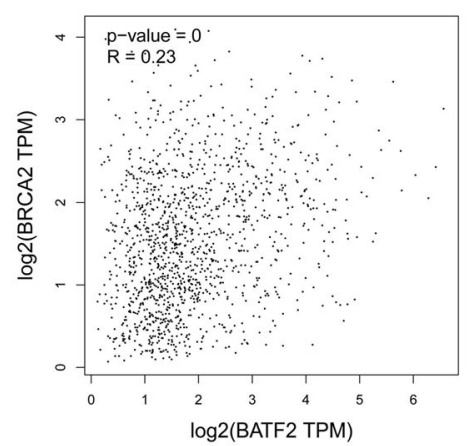

G

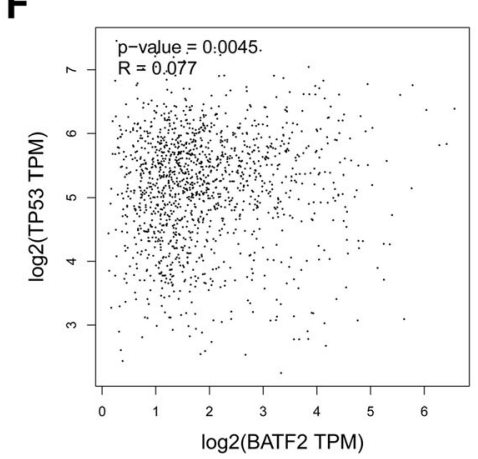

I
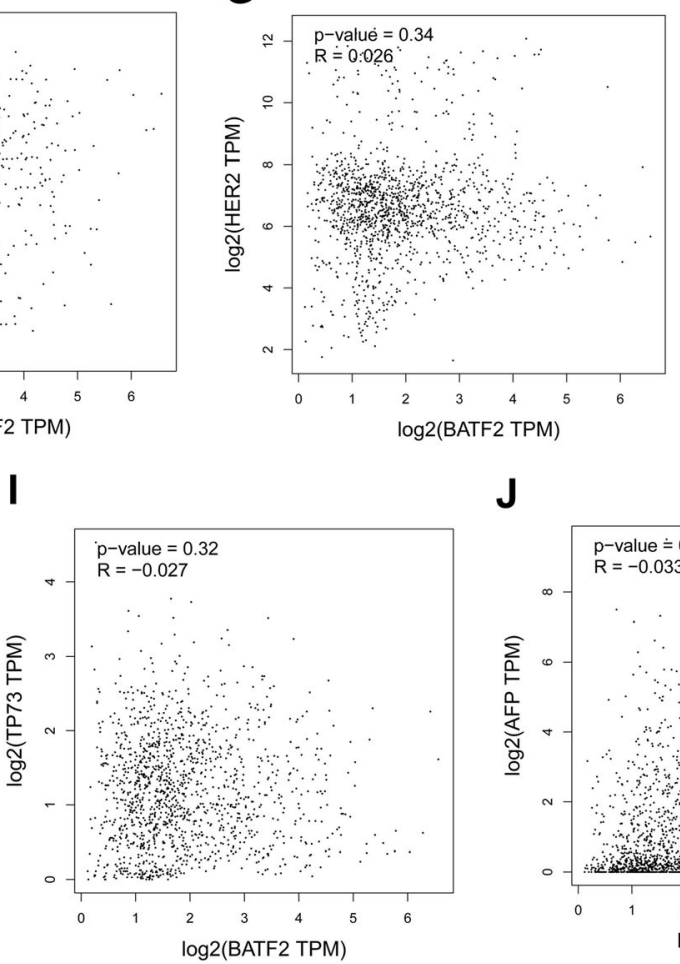

J
B

\begin{tabular}{|c|c|c|c|}
\hline Query & Gene & Gene Symbol and Name & Score \\
\hline BATF2 & STAT1 & $\begin{array}{r}\text { signal transducer and } \\
\text { activator of transcription 1 }\end{array}$ & 1.747 \\
\hline BATF2 & TAP1 & $\begin{array}{r}\text { transporter 1, ATP-binding } \\
\text { cassette, sub-family B } \\
\text { (MDR/TAP) }\end{array}$ & 1.403 \\
\hline BATF2 & PSMB9 & $\begin{array}{r}\text { proteasome subunit beta 9 } \\
\text { PSM }\end{array}$ & 1.123 \\
\hline
\end{tabular}

E

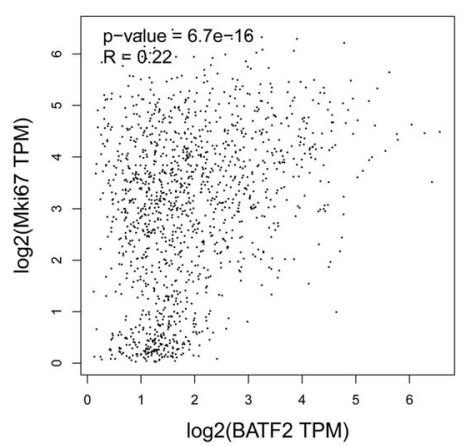

H

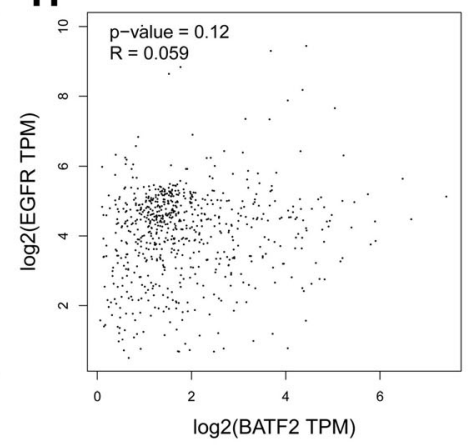

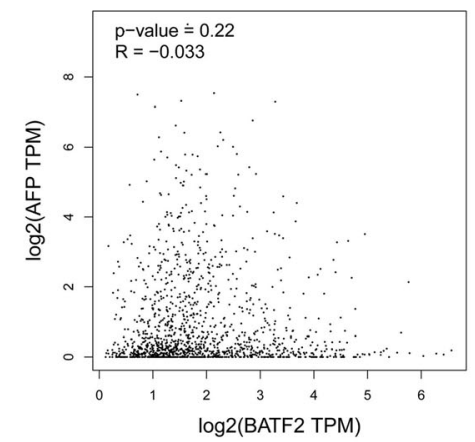

Fig. 4 Co-expression networks and correlation analysis of BATF2 utilized Coexpedia tool. (A) Co-expression networks of BATF2 in Pan-cancer. (B) Co-expression genes with BATF2 in BC. Correlation analysis of BATF2 mRNA with (C) AR, (D)BRCA2, (E)Mki67, (F)TP53, (G)HER2, (H) EGFR, (I)TP73, and (J) AFP based on GEPIA database 


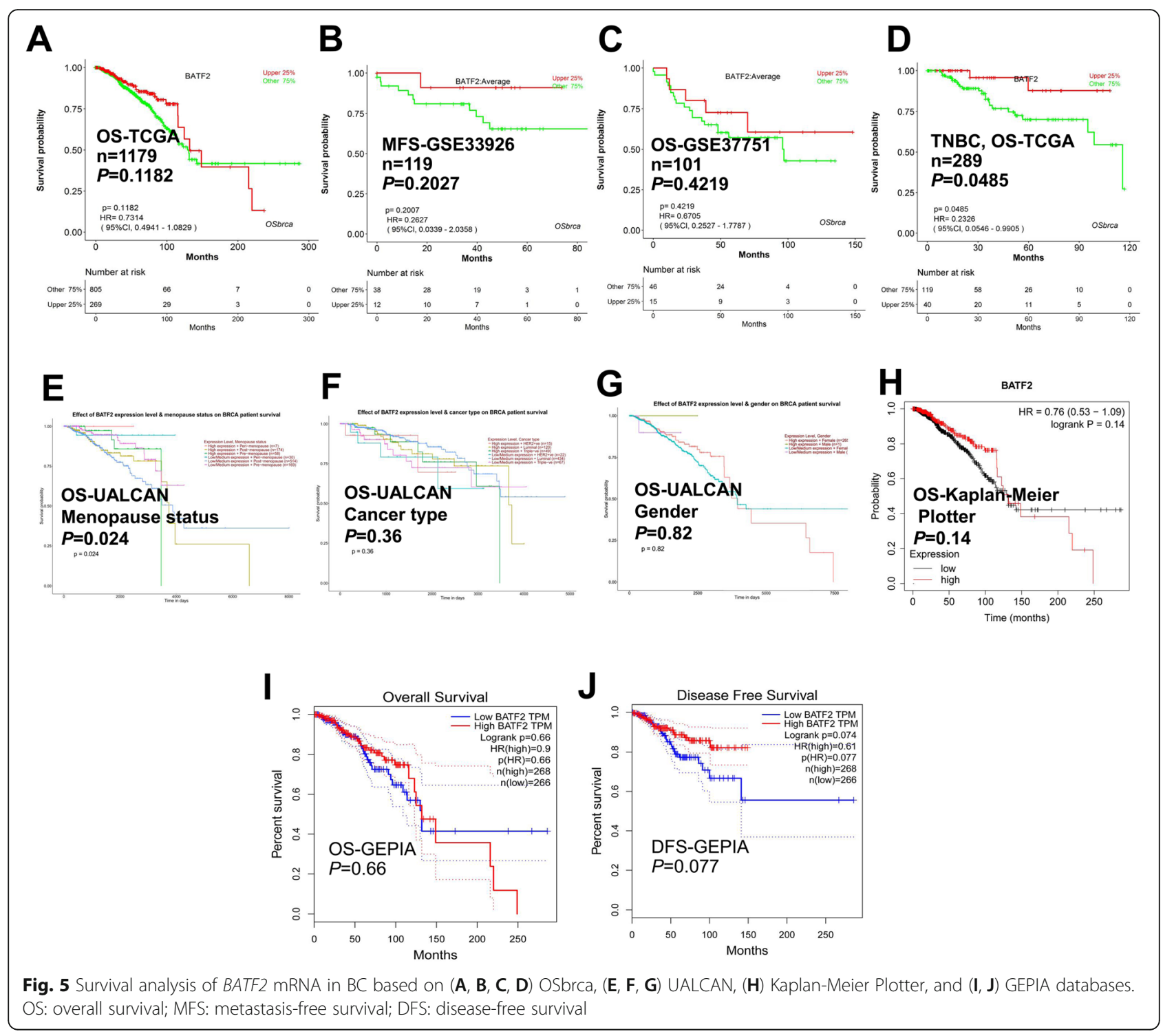

indicators of a better prognosis of BRCA patients (Fig. $5 \mathrm{E})$ (cancer type and gender were not significant indicators, see Fig. 5F and G). However, there were nonsignificant correlations between BATF2 mRNA expression and the survival of BRCA patients from KaplanMeier Plotter and GEPIA databases (Fig. 5H-J).

\section{IHC validation for the prognostic value of BATF2 protein expression in $\mathrm{BC}$}

We further detected BATF2 protein expression in cancerous and adjacent noncancerous tissues in $\mathrm{BC}$ using high-throughput tissue microarray data and validated the expression using IHC analysis. The results showed that BATF2 was mainly expressed in the nucleus of BC cancer cells (Fig. 6A). Of 145 patients, 68 were positive for BATF2 expression, with a positive rate of $46.90 \%$. Of the 68 positive individuals, only $19.17 \%(13 / 68)$ showed a moderate expression level $(+)$, and the remaining were with a low level (weak staining, \pm ). The positive rate of BATF2 expression in adjacent noncancerous tissues reached $62.22 \%$ (56/90). Besides, BATF2 expression (rating scores) were higher in patients with stage I-II than those with stage III (Fig. 6B). BATF2 protein expression was correlated with clinical stage $(P<0.0001$; Table 1$)$ and AR expression $(P=0.0393$; Fig. $6 C)$, rather than estrogen receptor (ER), progesterone receptor (PR), HER2, Mki67, TP53 and EGFR (all with $P>0.05$; Fig. 6C). Correlation analysis showed that BATF2 protein level was related to clinical stage $(\mathrm{OR}=4.295,95 \% \mathrm{CI}: 1.947-9.477$, $P=0.000$; Fig. 6D). The survival analysis showed that BATF2 protein expression was positively associated with $\mathrm{BC}$ prognosis: the OS of patients positive for BATF2 expression was significantly prolonged compared to those negative for BATF2 expression $(\mathrm{HR}=0.3303,95 \% \mathrm{CI}$ : 


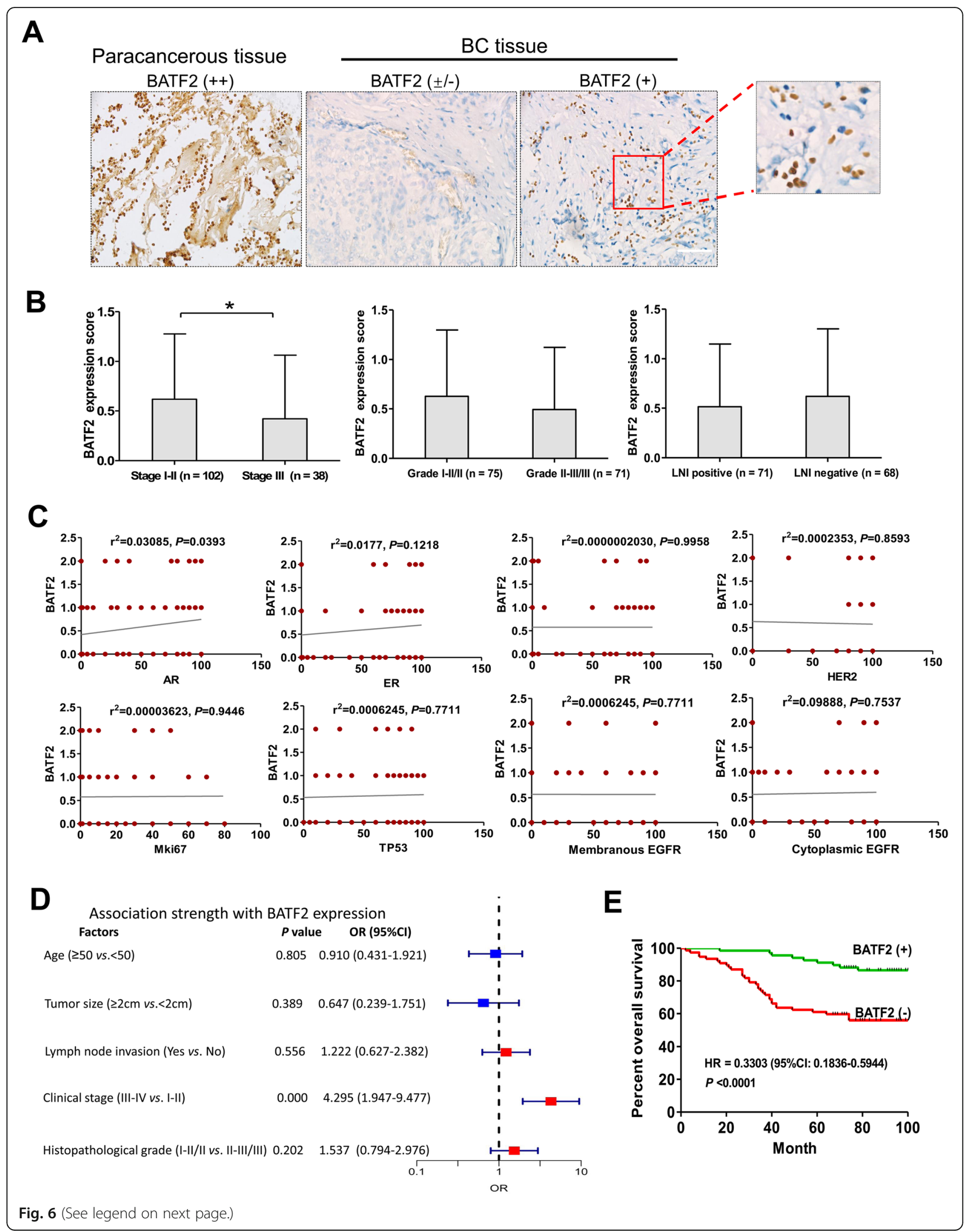


(See figure on previous page.)

Fig. 6 External validations of BATF2 protein expression in BC tissues by IHC. (A) Expression of BATF2 in BC and paracancerous tissues $(\times 200)$. (B) BATF2 level (rating score) in BC patients grouped by the status of clinical stage, pathology grade and lymph node invasion (LNI). (C) correlation analysis of BATF2 protein expression with AR, ER, PR, HER2, Mki67, TP53 and EGFR. (D) Analysis of the association strength between BATF2 expression and clinicopathological parameters in BC. (E) The plotted survival curve of BATF2 protein in predicting the OS of BC cases $(n=145) .{ }^{*} P<0.05$

0.1836-0.5944; $P<0.0001$; Fig. 6E). These results suggest that BATF2 can be used as a prognostic indicator of BC.

\section{Serum and exosomal BATF2 mRNA expressions in BC patients}

The morphology and size of serum-derived exosome vesicles were assessed using the TEM examination. TEM images revealed serum exosomes were tiny, $30-150 \mathrm{~nm}$ vesicles with a membrane structure (Fig. $7 \mathrm{~A}$ ). They were further identified by determining the expressions of exosomal marker proteins CD9 and CD63 using western blot analysis (Fig. 7B). CD9 and CD63 expressions were detectable in the eluent, whereas Cytochrome $\mathrm{C}$ expression was negative (a negative control to exclude the possible mixture of cellular contamination), indicating that serum exosomes were successfully extracted. The qRTPCR assay showed that the positive rates of serum and exosomal BATF2 mRNA expressions were $45.00 \%$ (27/ $60)$ and $41.67 \%(25 / 60)$ in BC patients, respectively, versus the positive rates of $57.14 \%(32 / 56)$ and $51.80 \%(29 /$ 56) in healthy controls. Serum BATF2 mRNA expressions were down-regulated in $\mathrm{BC}$ cases (Fig. $7 \mathrm{C}$ and $\mathrm{D}$ ). For serum BATF2 mRNA expression, ROC curves yielded a sensitivity of $84.38 \%$, a specificity of $85.19 \%$
(Youden index $=0.6956$ ), and the AUC of 0.8929 for BC diagnosis from healthy individuals (Fig. 7E). Similarly, exosomal BATF2 mRNA expressions were also decreased in BC cases (Fig. 7F and G). Exosomal BATF2 mRNA expression exhibited an AUC of 0.8869 for BC diagnosis (Fig. $7 \mathrm{H}$ ), with a sensitivity of $82.76 \%$ and a specificity of $80.00 \%$ (Youden index $=0.6275$ ). These results suggest that serum and exosomal BATF2 mRNA expressions are promising indicators for $\mathrm{BC}$ diagnosis.

\section{Discussion}

The occurrence of breast cancer (BC) is a femaleprevalent malignancy with massive involvement of intricate proto-oncogene networks and tumor suppressor gene inactivation [3-5]. Current evidence supports $B A T F 2$ as a tumor suppressor gene in various malignancies. Su ZZ et al. first reported that BATF2 overexpression in malignant glioma, melanoma, and prostatic cancer cell lines strongly inhibited the growth and apoptosis of cancer cells, without harm to the survival of noncancerous cells [6]. Ma $\mathrm{H}$ et al. found that the low BATF2 expression was positively correlated with the occurrence and development of liver cancer, and strikingly, all patients with down-regulated BATF2 expression had

Table 1 Correlations between BATF2 expressions and clinical characteristics in BC patients

\begin{tabular}{|c|c|c|c|c|c|}
\hline Clinicopathological features & Total cases & BATF2 expression (+) & $\begin{array}{l}\text { BATF2 } \\
\text { expression } \\
(-)\end{array}$ & $\begin{array}{l}\mathrm{X}^{2} \\
\text { value }\end{array}$ & $P$ value \\
\hline Age (years) & 145 & & & 0.061 & 0.805 \\
\hline$\geq 50$ & 108 & 50 & 58 & & \\
\hline$<50$ & 37 & 18 & 19 & & \\
\hline Tumor size & 141 & & & 0.743 & 0.389 \\
\hline$\geq 2 \mathrm{~cm}$ & 123 & 55 & 68 & & \\
\hline$<2 \mathrm{~cm}$ & 18 & 10 & 8 & & \\
\hline Lymph node invasion & 139 & & & 0.348 & 0.556 \\
\hline Yes & 69 & 34 & 35 & & \\
\hline No & 70 & 31 & 39 & & \\
\hline Clinical stage & 140 & & & 13.964 & $<0.0001$ \\
\hline Stage I+ II & 94 & 54 & 40 & & \\
\hline Stage III & 46 & 11 & 35 & & \\
\hline Histopathological grade & 143 & & & 1.631 & 0.202 \\
\hline$|-||/| \mid$ & 74 & 39 & 35 & & \\
\hline$\|-\mid\| /\|/\|$ & 69 & 29 & 40 & & \\
\hline
\end{tabular}




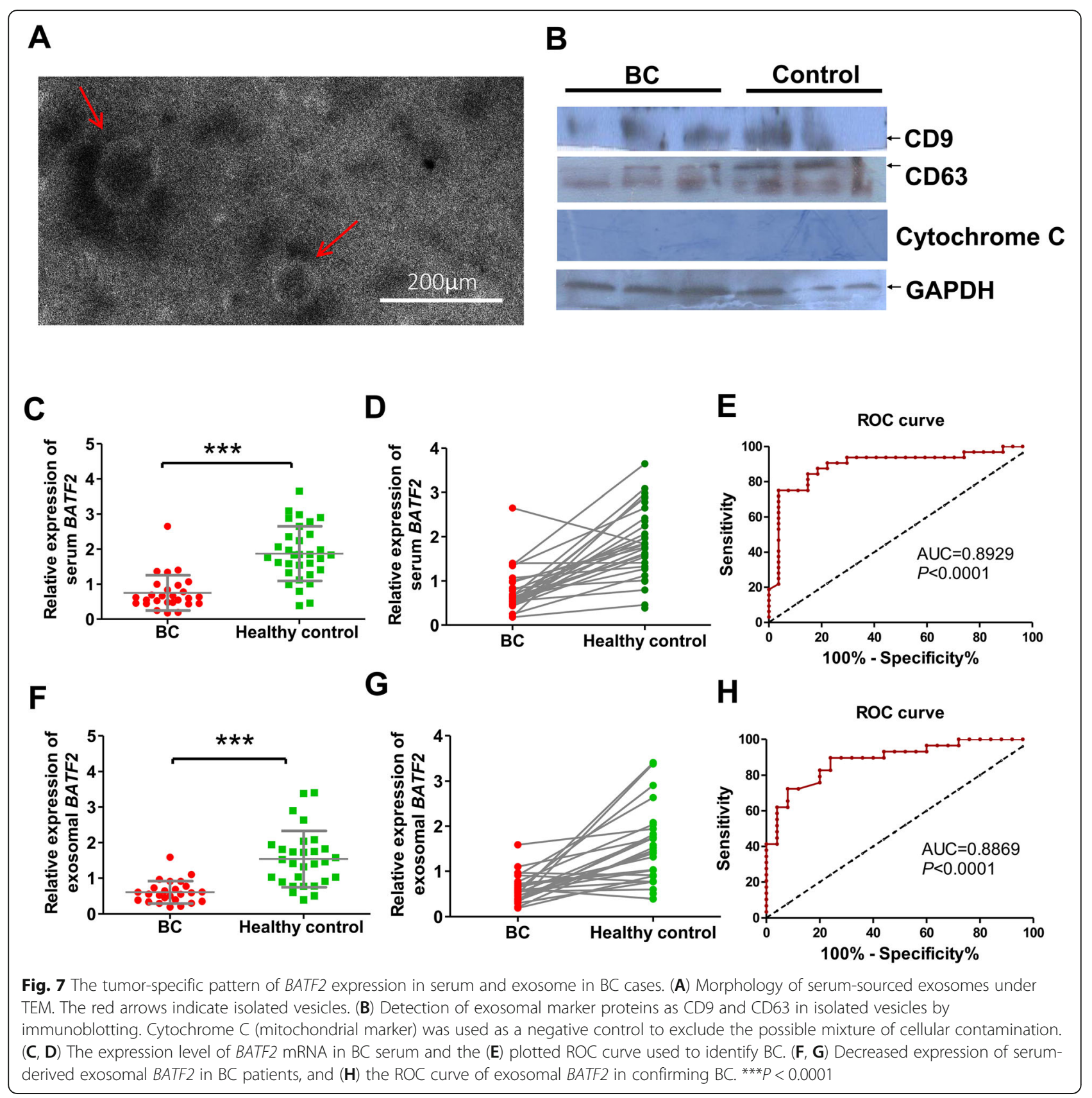

a poor prognosis [11]. Li et al. reported that BATF2 expression in prostate cancer was significantly associated with clinicopathological features such as serum PSA levels, clinical stage, and distant metastasis, which could be a critical player in the recurrence and progression of prostate cancer [26]. Consistently, other studies also suggest that a low BATF2 level is a risk factor for the poor prognosis in non-small cell lung cancer; BATF2 deletion promotes the EMT process, leading to LUAD cell invasion and metastasis [15]. BATF2 mRNA expression was also significantly down-regulated in cancerous tissues of colorectal cancer: patients negative for BATF2 protein expression often exhibit a poor grade of tumor differentiation, deep invasion, a higher TNM-stage, and a short period of postoperative survival, with significant correlations [10]. The study of CML showed that CML patients often had lower serum BATF2 mRNA expression levels than healthy individuals; the down-regulation of $B A T F 2$ gene expression is related to $B C R-A B L$ inhibition and participates in the occurrence and development of CML [8]. These mentioned studies imply that BATF2 can be used as a prognostic indicator of patients, a monitoring sensor for tumor therapy, and a potential target in gene therapy. 
This work initially assessed BATF2 mRNA and protein expressions as diagnostic and prognostic biomarkers in $\mathrm{BC}$ using bioinformatics. Subsequently, these expressions and their clinical implications were fully confirmed in the serum, exosome, and cancer tissue samples of $\mathrm{BC}$ patients using qRT-PCR and IHC analyses. In the first step, we comprehensively analyzed BATF2 mRNA and protein expressions using the expression data from GEPIA and UALCAN and HPA data from TCGA and GTEX. We found the expressions of BATF2 mRNA and protein in $\mathrm{BC}$ tissues were at low-to-moderate levels. The average expression level of BATF2 mRNA in healthy controls was slightly higher than that in $\mathrm{BC}$ cancer tissues, but there was no statistical difference. By contrast, BATF2 protein was mainly located in the nucleus of BC cancer cells based on HPA analysis, with a low-to-moderate level in protein expressions in 7 cases out of 12 cases. The correlation analysis revealed that BATF2 mRNA was co-expressed with TAP1, STAT1, and $P S M B 9$ in BC. Based on the GEPIA database, $B A T F 2$ mRNA expression was negatively associated with the AR expression and positively correlated with $B R C A 2$, $M k i 67$, and TP53 expressions, with non-significant relationships with HER2, EGFR, AFP and TP73 expressions. Some studies have confirmed that BATF2 expression is negatively correlated with CCN1 expression and regulates the biological behaviors of cancer cells via regulating $\mathrm{CCN} 1$ expression in vivo [9].

We further evaluated the relationship between the differentially expressed BATF2 mRNA levels and BC prognosis in UALCAN [19], OSbrca [21], Kaplan-Meier Plotter [22], GEPIA [18], and other databases. Most databases yielded a non-significant correlation between BATF2 mRNA expression and the prognosis, but the subgroup analyses uncovered the significantly prolonged OS of TNBC patients with high BATF2 mRNA expressions versus the low expression group. BATF2 mRNA expression levels and menopause status were also associated with the survival of BRCA patients. However, information on BATF2 expression and other biomarkers for $\mathrm{BC}$ prognosis is currently needed. In external validations, we determined the expression and prognostic value of BATF2 in BC patients using tissue microarray and IHC analysis. The results showed that BATF2 was mainly located in the nucleus of cancer cells of $\mathrm{BC}$, which was consistent with the results of HPA analysis, yet with a lower positive rate of BATF2 protein expression. And all validation samples positive for BATF2 protein showed its expressions at low-to-moderate levels (according to the IHC staining score). Correlations analysis showed that BATF2 protein expression was positively correlated with AR expression, which is in line with the correlation analysis results based on GEPIA. The survival analysis based on tissue microarray data showed that patients with high BATF2 expressions had a longer OS. Therefore, a high BATF2 expression in BC can be a protective factor for the prognosis of patients. However, the survival analysis of BATF2 expressions in HPA database showed that BATF2 expression (high $=254$ versus low $=$ 821 ) yielded a $P$ value of 0.053 in predicting the OS of the breast invasive carcinoma patients. Further investigations are still needed to verify the prognostic significance of BATF2 in BC.

Exosomes are extracellular nanovesicles $(30-150 \mathrm{~nm})$ fabricated via a series of regulatory processes, as simplified by "endocytosis - fusion - exocytosis" [26]. Recent studies have shown that exosomes act as carriers containing miRNA, mRNA, DNA fragments, proteins, and other bioactive substances, involving in various physiological and pathological processes [27, 28]. It has been proven that exosomes are enriched in the peripheral blood, urine, saliva, ascites, amniotic fluid, and other body fluids; and that tumor-derived or tumor-related exosomes even participate in the regulation of tumor occurrence and development [29]. The quantification of tumor exosomes can assist in early diagnosis, curative effect evaluation, and the prognosis of tumor patients [30]. Our previous studies reported that the clinical implications of serum and exosomal $L D H C$ gene (a CTA molecule) expressions in $\mathrm{BC}$ and $\mathrm{HCC}$, serving as an assistant for diagnosis, efficacy evaluation, and recurrence monitoring [23, 24]. In $B C$ patients, the positive rates of BATF2 mRNA expressions in the serum and exosomes were 45.00 and $41.67 \%$, respectively, versus 57.14 and $51.80 \%$ in healthy controls. Both serum and exosomal BATF2 showed the AUCs for BC diagnosis of higher than 0.85 , which exhibited promising diagnostic values. Similarly, Roe JK et al. reported the BATF2 transcript level as a single sensitive biomarker in differentiating active pulmonary and extracellular TB from healthy individuals [30]. Our study, for the first time, confirmed the expression and diagnostic value of serum and exosomal BATF2 in $\mathrm{BC}$, providing preliminary evidence for further research on the clinical application of BATF2 in $\mathrm{BC}$ patients.

Besides, we discovered that the prognosis assessment based on BATF2 tissue microarray did not yield the results of BATF2 mRNA as a molecular index for $\mathrm{BC}$ prognosis prediction. Several explanations can be considered. Firstly, the survival analysis was conducted based on BATF2 protein expressions using IHC scores. Secondly, BATF2 mRNA expression levels can be inconsistent with its protein levels due to protein posttranslation modifications, and different analyses for protein and mRNA expressions may also contribute to inconsistent results. It is reported that the linear relationship between mRNA and protein expression levels is only about 0.4 to 0.5 . For instance, a study by Antonis Koussounadis et al. 
reported that there was merely a weak correlation between all their measured mRNA and protein expression levels $(r=0.08, n=579, P=0.07)$ [31]. Thus, different measurement analyses, statistical methods, and expression levels cohesively result in inconsistent results.

\section{Conclusions}

This study demonstrates the down-regulation of BATF2 mRNA and protein expressions in $\mathrm{BC}$ and their diagnostic and prognostic implications in part of $\mathrm{BC}$ subtypes based on bioinformatics. Further clinical validations using serum and exosome samples have confirmed the results, which suggest that BATF2 is expected to be a new molecular marker for $\mathrm{BC}$ diagnosis and prognosis assessment. However, the sample size in this study is insufficient, which may inevitably bias the conclusion to some extent. Therefore, more validations incorporating large sample data are needed to further confirm the findings in this study.

\begin{abstract}
Abbreviations
AR: Androgen receptor; BATF2: Basic leucine zipper ATF-like transcription factor 2; BC: Breast cancer; BRCA2: BRCA2 DNA repair associated; CCN1: Cellular communication network factor 1; CML: Chronic myeloid leukemia; ESCC: Esophageal squamous cell carcinoma; EMT: Epithelial-mesenchymal transition; HPA: Human Protein Atlas; HCC: Hepatocellular carcinoma; IHC: Immunohistochemistry; IFN: Type I interferon; LUAD: Lung adenocarcinoma; Mki67: Marker of proliferation Ki-67; NSCLC: Non-small-cell lung cancer; OS: Overall survival; qRT-PCR: Real-time quantitative reverse transcription polymerase chain reaction; TP53: Tumor protein p53; TNBC: Triple-negative breast cancer
\end{abstract}

\section{Supplementary Information}

The online version contains supplementary material available at https://doi. org/10.1186/s12885-021-08785-6.

Additional file 1.

\section{Acknowledgements}

Not applicable.

\section{Authors' contributions}

ZLC, YC and XFW designed the study; YYL, XSZ and WP collected the literature and conducted the analysis of pooled data; $Y Y L$ wrote the manuscript; WP and JW helped to draft the manuscript; ZLC, YC and XFW proofread, revised and final approved the manuscript; all authors have approved the version to be published.

\section{Funding}

This study was supported by the National Natural Science Foundation of China (Grant number: 81802631), Joint Funds for the Innovation of Science and Technology, Fujian province (Grant number: 2017Y9072), and Science and Technology Program of Fujian Province, China (Grant number: 2018Y2003). The funding bodies played no role in the design of the study and collection, analysis, and interpretation of data and in writing the manuscript.

\section{Availability of data and materials}

The datasets used and/or analyzed during the current study are available from the corresponding author upon reasonable request.

\section{Declarations}

\section{Competing interest}

The authors declare that they have no competing interests.

\section{Ethics approval and consent to participate}

The study was approved by the Ethics Committee of Fujian Medical University Cancer Hospital (ethical approval certificate: No. SQ2018-015-01), and the study was conducted in compliance with the principle of the Declaration of Helsinki. Written informed consent was obtained from all patients or their family members.

\section{Consent for publication}

Not applicable.

Received: 28 November 2020 Accepted: 15 September 2021 Published online: 26 September 2021

\section{References}

1. DeSantis C, Siegel R, Bandi $P$, Jemal A. Breast cancer statistics, 2011. CA Cancer J Clin. 2011;61(6):409-18. https://doi.org/10.3322/caac.20134.

2. Chen W, Zheng R, Baade PD, Zhang S, Zeng H, Bray F, et al. Cancer statistics in China, 2015. CA Cancer J Clin. 2016;66(2):115-32. https://doi.org/10.3322/ caac.21338.

3. Coughlin SS. Epidemiology of breast Cancer in women. Adv Exp Med Biol. 2019:1152:9-29. https://doi.org/10.1007/978-3-030-20301-6_2.

4. McCuaig JM, Stockley TL, Shaw P, Fung-Kee-Fung M, Altman AD, Bentley J, et al. Evolution of genetic assessment for BRCA-associated gynaecologic malignancies: a Canadian multisociety roadmap. J Med Genet. 2018;55(9): 571-7. https://doi.org/10.1136/jmedgenet-2018-105472.

5. Winters S, Martin C, Murphy D, Shokar NK. Breast Cancer epidemiology, prevention, and screening. Prog Mol Biol Transl Sci. 2017;151:1-32. https:// doi.org/10.1016/bs.pmbts.2017.07.002.

6. Su ZZ, Lee SG, Emdad L, Lebdeva IV, Gupta P, Valerie K, et al. Cloning and characterization of SARI (suppressor of AP-1, regulated by IFN). Proc Natl Acad Sci U S A. 2008;105(52):20906-11. https://doi.org/10.1073/pnas. 0807975106.

7. Murphy TL, Tussiwand R, Murphy KM. Specificity through cooperation: BATF-IRF interactions control immune-regulatory networks. Nat Rev Immunol. 2013;13(7):499-509. https://doi.org/10.1038/nri3470

8. Huang $Q$, Yang $Y$, Li X, Huang S. Transcription suppression of SARI (suppressor of AP-1, regulated by IFN) by BCR-ABL in human leukemia cells. Tumour Biol. 2011;32(6):1191-7. https://doi.org/10.1007/s13277-011-0222-1.

9. Dash R, Su ZZ, Lee SG, Azab B, Boukerche H, Sarkar D, et al. Inhibition of AP1 by SARI negatively regulates transformation progression mediated by CCN1. Oncogene. 2010;29(31):4412-23. https://doi.org/10.1038/onc.2010.194.

10. Liu ZB, Yang $Y$, Ye $X G$, Wang $L$, Tian $P Y$, Zhang $Y Y$. Expression and significance of SARI and CCN1 in human colorectal carcinomas. Zhonghua Yi Xue Za Zhi. 2011;91(34):2397-401.

11. Ma H, Liang $X$, Chen $Y$, Pan $K$, Sun J, Wang $H$, et al. Decreased expression of BATF2 is associated with a poor prognosis in hepatocellular carcinoma. Int J Cancer. 2011;128(4):771-7. https://doi.org/10.1002/ijc.25407.

12. Zhou RJ, Shi Z, Zhou K, Wang HD, Zhang GQ, Li XT, et al. Decreased SARI expression predicts poor prognosis of Chinese patients with non-small cell lung cancer. Int J Clin Exp Pathol. 2013;6(10):2056-63.

13. Han T, Wang Z, Yang Y, Shu T, Li W, Liu D, et al. The tumor-suppressive role of BATF2 in esophageal squamous cell carcinoma. Oncol Rep. 2015;34(3): 1353-60. https://doi.org/10.3892/or.2015.4090.

14. Xie JW, Huang XB, Chen QY, Ma YB, Zhao YJ, Liu LC, et al. m (6) a modification-mediated BATF2 acts as a tumor suppressor in gastric cancer through inhibition of ERK signaling. Mol Cancer. 2020;19(1):114. https://doi. org/10.1186/s12943-020-01223-4

15. Wang C, Su Y, Zhang L, Wang M, You J, Zhao X, et al. The function of SARI in modulating epithelial-mesenchymal transition and lung adenocarcinoma metastasis. PLoS One. 2012;7(9):e38046. https://doi.org/10.1371/journal.pone. 0038046.

16. Desany B, Zhang Z. Bioinformatics and cancer target discovery. Drug Discov Today. 2004;9(18):795-802. https://doi.org/10.1016/S1359-6446(04)03224-6.

17. Gómez-López G, Valencia A. Bioinformatics and cancer research: building bridges for translational research. Clin Transl Oncol. 2008;10(2):85-95. https://doi.org/10.1007/s12094-008-0161-5. 
18. Tang Z, Li C, Kang B, Gao G, Li C, Zhang Z. GEPIA: a web server for cancer and normal gene expression profiling and interactive analyses. Nucleic Acids Res. 2017;45(W1):W98-w102. https://doi.org/10.1093/nar/gkx247.

19. Chandrashekar DS, Bashel B, Balasubramanya SAH, Creighton CJ, PonceRodriguez I, Chakravarthi B, et al. UALCAN: A Portal for Facilitating Tumor Subgroup Gene Expression and Survival Analyses. Neoplasia (New York, NY). 2017;19(8):649-58.

20. Yang S, Kim CY, Hwang S, Kim E, Kim H, Shim H, et al. COEXPEDIA: exploring biomedical hypotheses via co-expressions associated with medical subject headings (MeSH). Nucleic Acids Res. 2017;45(D1):D389d396. https://doi.org/10.1093/nar/gkw868.

21. Yan Z, Wang Q, Sun X, Ban B, Lu Z, Dang Y, et al. OSbrca: a web server for breast Cancer prognostic biomarker investigation with massive data from tens of cohorts. Front Oncol. 2019;9:1349. https://doi.org/10.3389/fonc.2019. 01349.

22. Nagy Á, Munkácsy G, Györffy B. Pancancer survival analysis of cancer hallmark genes. Sci Rep. 2021;11(1):6047. https://doi.org/10.1038/s41598021-84787-5.

23. Cui Z, Chen Y, Hu M, Lin Y, Zhang S, Kong L, et al. Diagnostic and prognostic value of the cancer-testis antigen lactate dehydrogenase $\mathrm{C} 4$ in breast cancer. Clin Chim Acta. 2020;503:203-9.

24. Cui Z, Li Y, Gao Y, Kong L, Lin Y, Chen Y. Cancer-testis antigen lactate dehydrogenase $C 4$ in hepatocellular carcinoma: a promising biomarker for early diagnosis, efficacy evaluation and prognosis prediction. Aging. 2020; 12(19):19455-67. https://doi.org/10.18632/aging.103879.

25. Lötvall J, Hill AF, Hochberg F, Buzás El, Di Vizio D, Gardiner C, et al. Minima experimental requirements for definition of extracellular vesicles and their functions: a position statement from the International Society for Extracellular Vesicles. J Extracell Vesicles. 2014;3(1):26913. https://doi.org/1 0.3402/jev.v3.26913.

26. Cocucci E, Meldolesi J. Ectosomes and exosomes: shedding the confusion between extracellular vesicles. Trends Cell Biol. 2015;25(6):364-72. https:// doi.org/10.1016/j.tcb.2015.01.004

27. Safdar A, Tarnopolsky MA. Exosomes as Mediators of the Systemic Adaptations to Endurance Exercise. Cold Spring Harb Perspect Med. 2018; 8(3):a029827.

28. Trotta T, Panaro MA, Cianciulli A, Mori G, Di Benedetto A, Porro C. Microgliaderived extracellular vesicles in Alzheimer's disease: a double-edged sword. Biochem Pharmacol. 2018;148:184-92. https://doi.org/10.1016/j.bcp.2017.12.020.

29. Qin J, Xu Q. Functions and application of exosomes. Acta Pol Pharm. 2014 71(4):537-43. https://doi.org/10.1024/0040-5930/a000549.

30. Roe JK, Thomas N, Gil E, Best K, Tsaliki E, Morris-Jones S, et al. Blood transcriptomic diagnosis of pulmonary and extrapulmonary tuberculosis. JCl Insight. 2016;1(16):e87238. https://doi.org/10.1172/jci.insight.87238.

31. Koussounadis A, Langdon SP, Um IH, Harrison DJ, Smith VA. Relationship between differentially expressed mRNA and mRNA-protein correlations in a xenograft model system. Sci Rep. 2015;5(1):10775. https:/doi.org/10.1038/ srep10775.

\section{Publisher's Note}

Springer Nature remains neutral with regard to jurisdictional claims in published maps and institutional affiliations.

Ready to submit your research? Choose BMC and benefit from:
- fast, convenient online submission
- thorough peer review by experienced researchers in your field
- rapid publication on acceptance
- support for research data, including large and complex data types
- gold Open Access which fosters wider collaboration and increased citations
- maximum visibility for your research: over 100M website views per year
At BMC, research is always in progress.
Learn more biomedcentral.com/submissions

